\title{
Daily sleep duration and risk of metabolic syndrome among middle-aged and older Chinese adults: cross-sectional evidence from the Dongfeng-Tongji cohort study
}

Jing $\mathrm{Wu}^{1,2}$, Guiqiang $\mathrm{X} \mathrm{u}^{1,2}$, Lijun Shen 1,2, Yanmei Zhang ${ }^{1,2}$, Lulu Song ${ }^{1,2}$, Siyi Yang ${ }^{1,2}$, Handong Yang ${ }^{3}$, Yuan Liang ${ }^{4}$, Tangchun $\mathrm{Wu}^{1}$ and Youjie Wang ${ }^{1,2^{*}}$

\begin{abstract}
Background: Evidence from epidemiological studies has demonstrated that a shorter or longer duration of nighttime sleep may increase the risk of metabolic syndrome. Little is known about the association between daily sleep duration, including nighttime sleep and daytime napping duration, and metabolic syndrome. We aimed to examine the association between daily sleep duration and metabolic syndrome and its components in middle-aged and older Chinese adults using data from the Dongfeng-Tongji Cohort study.

Methods: A total of 25,184 participants (mean age 63.6 years) who completed the baseline questionnaire, physical examination and laboratory tests were included in this analysis. Daily sleep duration was calculated by summing up nighttime sleep duration and daytime napping duration. The metabolic syndrome was defined using the International Diabetes Federation criteria. Logistic regression models were used to investigate the association between daily sleep duration and the risk of metabolic syndrome and its components.

Results: Of the participants, 8,046 (31.9\%) had metabolic syndrome. Females had a higher prevalence (38.6\%) of metabolic syndrome than males (23.9\%). Female participants with longer daily sleep duration ( $\geq 8$ hours, all $P<0.05)$ per day had a higher risk of metabolic syndrome compared with those sleeping 7-7.9 hours, adjusting for potential confounders. Longer daily sleep was positively associated with individual components of metabolic syndrome except central obesity in females, and was only positively associated with HDL-C in males. Further analysis revealed that a longer duration of daytime napping ( $\geq 90$ minutes, $P<0.05$ ) was associated with the risk of metabolic syndrome in females. However, nighttime sleep duration was not associated with the risk of metabolic syndrome in either males or females.
\end{abstract}

Conclusions: Our findings suggested that longer daytime napping duration rather than nighttime sleeping duration was associated with increased risk of metabolic syndrome in females. The findings have significant implications for further studies to explore the appropriate sleep duration for middle-aged and older adults.

Keywords: Metabolic syndrome, Daily sleep duration, Daytime napping duration, Nighttime sleep duration

\footnotetext{
* Correspondence: wangyoujie@mails.tjmu.edu.cn

${ }^{1}$ MOE Key Lab of Environment and Health, School of Public Health, Tongji

Medical College, Huazhong University of Science \& Technology, Hangkong

Road 13, Wuhan 430030, Hubei, China

${ }^{2}$ Department of Maternal and Child Health, School of Public Health, Tongji

Medical College, Huazhong University of Science \& Technology, Hangkong

Road 13, Wuhan 430030, Hubei, China

Full list of author information is available at the end of the article
}

(c) 2015 Wu et al.; licensee BioMed Central. This is an Open Access article distributed under the terms of the Creative Commons Attribution License (http://creativecommons.org/licenses/by/4.0), which permits unrestricted use, distribution, and reproduction in any medium, provided the original work is properly credited. The Creative Commons Public Domain Dedication waiver (http://creativecommons.org/publicdomain/zero/1.0/) applies to the data made available in this article, unless otherwise stated. 


\section{Background}

Metabolic syndrome (MetS), a clustering of physiologically related cardiovascular risk factors, including central adiposity, dyslipidemia, elevated blood pressure and increased glucose level, is a critical public health problem worldwide [1]. The syndrome is closely correlated with the development of chronic kidney disease, cardiovascular disease, and a higher risk of all-cause mortality [2-4]. In China, the age-standardized prevalence of MetS was $9.8 \%$ in males and $17.8 \%$ in females [5]. Given high prevalence of MetS, it is significant to identify modifiable risk factors to prevent MetS.

Sleep is a basic need for human, and closely related to the body's metabolism. Previous studies have indicated that short or long sleep duration is associated with an increased risk for MetS, but the findings are not consistent [6]. Studies have demonstrated that older adults had different sleep pattern compared with younger adults $[7,8]$. A meta-analysis performed by Flyd et al. found that sleep duration increased with age while sleep disturbances also increased [7], suggesting that older adults may have more daily sleep duration throughout the day. However, to date, there is little evidence about the effects of daily sleep duration on human health outcomes for older adults.

Daytime napping is traditionally regarded as a healthy life behavior and is especially good for older adults in China. The percentage of older Chinese adults who practice habitual daytime napping reaches $61.7 \%$ in men and $46.8 \%$ in women [9]. Accumulative evidence showed that daytime napping might be a marker of underlying health risks. A large cohort in British revealed that excessive daytime napping was associated with higher risk of mortality from all-cause and respiratory diseases [10]. $\mathrm{Xu}$ et al. demonstrated an independent association between longer durations of daytime sleep and the risk of diabetes in older adults [11]. Given the high prevalence of daytime napping for older adults in China, it is of great importance to examine the effects of daytime napping for health outcomes.

Evidence from studies have found different effects of daytime napping and nighttime sleep for health $[11,12]$, therefore, in this study, we aimed to examine the associations between daily sleep duration, including daytime napping and nighttime sleep with MetS in a Chinese middle-aged and older population based on the data from the Dongfeng-Tongji cohort study of retired workers.

\section{Methods}

\section{Study participants}

Data for our analysis was derived from the DongfengTongji Cohort study (DFTJ cohort) which was initiated in 2008 among retired employees of Dongfeng Motor Corporation (DMC) in Shiyan City, Hubei Province. The rationale, design, and methods of the DFTJ cohort have previously been described in detail [13]. Between 2008 and $2010,87 \%(27009 / 31000)$ of the invited retired employees responded to the study and completed baseline information, medical examinations and provided blood samples. For current analysis, the participants who had missing data on sleep habits or the determination of the status of MetS were excluded. Thus, the final sample included 25184 subjects. The demographic characteristics including sex and age were similar between individuals included in the current study and those excluded. This study was approved by the Medical Ethics Committee of the School of Public Health, Tongji Medical College, Huazhong University of Science and Technology and Dongfeng General Hospital, DMC. All participants signed an informed consent form.

\section{Assessment of MetS}

We classified participants as having MetS if they have central adiposity (defined as waist circumference $\geq 90 \mathrm{~cm}$ for Chinese males and $\geq 80 \mathrm{~cm}$ for Chinese females) plus any two of the following four factors according to the new International Diabetes Federation (IDF) definition [1]: a. triglycerides (TG) $\geq 1.7 \mathrm{mmol} / \mathrm{L}$ or specific treatment for this lipid abnormality; b. high-density lipoprotein cholesterol (HDL-C) $<1.03 \mathrm{mmol} / \mathrm{L}$ in males or $<1.29 \mathrm{mmol} / \mathrm{L}$ in females or specific treatment for this lipid abnormality; c. systolic blood pressure (SBP) $\geq 130 \mathrm{mmHg}$ or diastolic blood pressure (DBP) $\geq 85 \mathrm{~mm} \mathrm{Hg}$ or treatment of previously diagnosed hypertension; d. fasting plasma glucose (FPG) $\geq$ $5.6 \mathrm{mmol} / \mathrm{L}$ or previously diagnosed type 2 diabetes.

\section{Assessment of nighttime sleep, daytime napping and daily sleep}

Nighttime sleep duration was assessed by asking: "On average, how many hours and minutes do you sleep per night?". Nighttime sleep duration was classified as $<7$ hours, 7-7.9 hours, $8-8.9$ hours, and $\geq 9$ hours. Habitual daytime nappers were defined as those who had taken a planned or regular nap as a habit more than three times per week after lunch over the past twelve months. Daytime napping was assessed by asking "Do you have a habit of taking a nap after lunch?" Those who reported "Yes" were further asked about the average duration of their naps. Daytime napping duration was categorized as no daytime napping, $<30$ minutes, 3059 minutes, $60-89$ minutes, and $\geq 90$ minutes. Additionally, we computed daily sleep duration by summing up nighttime sleep duration and daytime napping duration, and we categorized it into five categories: $<7$ hours, $7-7.9$ hours, 8-8.9 hours, and $9-9.9$ hours and $\geq 10$ hours.

\section{Assessment of covariates}

Demographic information on age, sex, education level (elementary or below, junior high school, high school, 
college or above), and marital status (e.g., married, unmarried, widowed, divorced) was included in the questionnaire. History of physician-diagnosed chronic diseases and history of medication were also obtained. The criteria for family history of diabetes or hypertension were having at least one first-degree relative with a diagnosis of diabetes or hypertension. Health-related habits on smoking status, alcohol drinking status, and physical activity was also obtained. Physical activity was defined as those who exercise more than 20 min per day and more than three times per week over the last six months. The anthropometric and laboratory data included weight, height, waist circumference, systolic and diastolic blood pressure, high density lipoprotein, and triglyceride. Body mass index was calculated as weight in kilograms divided by height in meters squared.

\section{Statistical analysis}

Numerical variables were summarized as mean \pm SD and categorical data were presented as proportion (\%). Student $t$-tests were used to examine difference in means for the numerical data between participants with MetS and those without MetS, and Chi-square tests were used for categorical data. According to several studies, the prevalence of MetS displayed a gender difference that females have a higher prevalence of MetS than males [5,14]. Moreover, females reported to have more sleep disturbances than males in older adults [15]. Therefore, we conducted a gender-stratified analysis. Logistic regression analyses were used to estimate the odds ratios (ORs) and 95\% confidence intervals (95\% CIs) of daily sleep duration with MetS in males and females, respectively. We used participants with daily sleep duration of 7-7.9 hours as the reference group. Model 1 was adjusted for demographic factors (age, marriage, education), heath-related life behaviors (smoking status, drinking status, physical activity), self-reported physiciandiagnosed chronic diseases and a family history of diabetes or hypertension. Model 2 was adjusted for the variables in Model 1 plus BMI. Moreover, to better understand and interpret the results of the association between daily sleep duration groups and MetS, we further explored the effects of daytime napping duration or nighttime sleep duration on MetS by performing logistic regression analyses adjusting for covariates similar to the above-mentioned models. We used participants with nighttime sleep duration of 7-7.9 hours and those who did not nap in the daytime as references respectively in examining the associations among nighttime sleep duration and daytime napping duration with MetS. In addition, multivariable logistic regression was also used to test the relationship between daily sleep duration and the components of MetS in males and females, respectively. All statistical analysis was performed using
SPSS software (version 11.0). Associations were considered statistically significant at $P<0.05$.

\section{Results}

Of the 25,184 participants (11,370 males, 13,814 females), 8,046 (31.9\%) had MetS, females had a higher prevalence $(38.6 \%)$ of metabolic syndrome than males (23.9\%). Participants with MetS were more likely to be

Table 1 The characteristics of study participants with and without the metabolic syndrome $(n=25,184)$

\begin{tabular}{|c|c|c|c|}
\hline & $\begin{array}{l}\text { Participants } \\
\text { with MetS }\end{array}$ & $\begin{array}{l}\text { Participants } \\
\text { without MetS }\end{array}$ & \\
\hline Characteristics & $(n=17,138)$ & $(n=8,046)$ & $P$ \\
\hline Age (year) & $64.6 \pm 7.7$ & $63.2 \pm 7.8$ & $<0.001$ \\
\hline Sex & & & $<0.001$ \\
\hline Male (\%) & $2720(33.8)$ & $8650(50.5)$ & \\
\hline Female (\%) & $5326(66.2)$ & $8488(49.5)$ & \\
\hline Marital status & & & $<0.001$ \\
\hline Married (\%) & $7025(87.5)$ & $15518(90.8)$ & \\
\hline Widowed (\%) & $845(10.5)$ & $1172(6.9)$ & \\
\hline Unmarried (\%) & $22(0.3)$ & $54(0.3)$ & \\
\hline Divorced (\%) & $133(1.7)$ & $350(2.0)$ & \\
\hline Education & & & $<0.001$ \\
\hline Elementary or below (\%) & $2789(34.9)$ & $4598(27.1)$ & \\
\hline Junior high school (\%) & $2922(36.6)$ & $6058(35.6)$ & \\
\hline High school (\%) & $1641(20.6)$ & $4362(25.7)$ & \\
\hline College or above (\%) & $630(7.9)$ & $1979(11.6)$ & \\
\hline Physical activity (\%) & $6454(80.2)$ & $14067(82.1)$ & $<0.001$ \\
\hline Current smoker (\%) & $1011(12.7)$ & $3496(20.5)$ & $<0.001$ \\
\hline Current drinker (\%) & $1275(15.9)$ & $3997(23.3)$ & $<0.001$ \\
\hline \multicolumn{4}{|l|}{ Self-reported medical history } \\
\hline Coronary heart disease (\%) & $1775(22.3)$ & $2121(12.5)$ & $<0.001$ \\
\hline Myocardial infarction (\%) & $278(3.5)$ & $423(2.5)$ & $<0.001$ \\
\hline Stroke (\%) & $443(5.6)$ & $605(3.6)$ & $<0.001$ \\
\hline Hypertension (\%) & $4623(57.5)$ & $5023(52.1)$ & $<0.001$ \\
\hline Diabetes mellitus (\%) & $1712(21.3)$ & $1383(8.1)$ & $<0.001$ \\
\hline Family history of diabetes (\%) & $470(6.0)$ & $64.4(5.1)$ & 0.004 \\
\hline Family history of hypertension (\%) & $2008(25.3)$ & $3498(20.8)$ & $<0.001$ \\
\hline Body mass index $\left(\mathrm{kg} / \mathrm{m}^{2}\right)$ & $27.0 \pm 3.1$ & $23.3 \pm 2.9$ & $<0.001$ \\
\hline Waist circumference (cm) & $91.0 \pm 7.5$ & $79.3 \pm 7.9$ & $<0.001$ \\
\hline Fasting plasma glucose (mmol/L) & $6.6 \pm 2.1$ & $5.8 \pm 1.5$ & $<0.001$ \\
\hline Systolic blood pressure (mmHg) & $136.1 \pm 18.0$ & $126.6 \pm 18.1$ & $<0.001$ \\
\hline Diastolic blood pressure $(\mathrm{mmHg})$ & $80.8 \pm 11.1$ & $76.3 \pm 10.5$ & $<0.001$ \\
\hline $\mathrm{HDL}-\mathrm{C}(\mathrm{mmol} / \mathrm{L})$ & $1.3 \pm 0.4$ & $1.5 \pm 0.4$ & $<0.001$ \\
\hline Triglycerides (mmol/L) & $5.3 \pm 1.0$ & $5.1 \pm 0.9$ & $<0.001$ \\
\hline
\end{tabular}

Data are presented as mean \pm SD or number (percentage).

Abbreviations: MetS metabolic syndrome, $H D L-C$ high-density lipoprotein cholesterol. 
non-smokers, nondrinkers, and had less physical activity (Table 1). A higher proportion of participants with MetS had physician diagnosed chronic diseases (all $P<0.001$ for listed diseases) compare with those without MetS. In addition, participants with MetS were more likely to have higher blood pressure, higher level of triglycerides, and higher body mass index.

Table 2 presents the prevalence of MetS in each sleep duration category by gender. Using Chi-square test, we found a higher prevalence of MetS in participants with longer duration of daily sleeping than those with daily sleep duration of 7-7.9 hours in females. There was statistically significance between different nighttime sleep groups and the prevalence of MetS in females. Moreover, female participants with longer duration of daytime napping had a higher prevalence of MetS compared with those who did not nap. Daily sleep duration, nighttime sleep duration and daytime duration had no effects on the prevalence of MetS in males.

The associations between duration of daily sleep and Mets stratified by genders were displayed in Table 3. Model 1 showed that females participants with a daily sleep duration of 8-8.9 hours (OR, 1.16 [95\% CI, 1.04-1.30]), 9-9.9 hours (OR, 1.22 [95\% CI, 1.08-1.36]), and $\geq 10$ hours (OR, 1.29 [95\% CI, 1.13-1.46]) had a significantly higher odds ratio (OR) for MetS compared with females with daily sleep duration of 7-7.9 hours after adjustment for demographic factors (age, marriage, education), heath-related life behaviors (smoking status, drinking status, physical activity), self- reported physician-diagnosed chronic diseases and a family history of diabetes or hypertension. In model 2, after adjusting for BMI which is a main known risk factor for MetS, the ORs were 1.20 (95\% CI, 1.06-1.37), 1.28 (95\% CI, 1.12-1.46), and 1.33 (95\% CI, 1.15-1.54) for females who slept 8-8.9 hours, 9-9.9 hours, $\geq 10$ hours, respectively, compared with females who slept 7-7.9 hours. To better understand and provide valuable clues to explain the relationship between daily sleep duration and MetS, we further analyzed nighttime sleep duration or daytime napping duration on the risk of MetS using a series of logistic regression models (Table 3). Nighttime sleep duration was not associated with MetS in all three models after adjusting for demographic factors, heath-related life behaviors, disease history, and daytime napping duration in either males or females. With regard to the association between daytime napping duration and MetS, model 1 showed that longer daytime napping durations of $\geq 90$ minutes (OR, 1.22 [95\% CI, 1.09-1.36]) had a risk of MetS compared with those who never napped in the daytime in females, while shorter napping time ( $<90$ minutes) had no effects on the risk of MetS (Table 3), after adjusting for demographic factors, heath-related life behaviors, self-reported physiciandiagnosed chronic diseases and a family history of diabetes or hypertension. Model 2 showed that longer napping duration of $\geq 90$ minutes (OR, 1.17 [95\% CI, 1.08-1.27]) still was associated with higher risk of MeSt after adjustment for BMI in females. When nighttime sleep duration was added in model 3, the risk of MetS was moderately

Table 2 Prevalence of metabolic syndrome by sleep duration stratified by gender

\begin{tabular}{|c|c|c|c|c|c|c|}
\hline & \multicolumn{2}{|l|}{ Male } & \multirow[t]{2}{*}{$P$} & \multicolumn{2}{|l|}{ Female } & \multirow[t]{2}{*}{$P$} \\
\hline & Non-MetS & MetS & & Non-MetS & MetS & \\
\hline Daily sleep duration (hours) & & & 0.12 & & & $<0.001$ \\
\hline$<7$ & $236(72.6)$ & $89(27.4)$ & & $370(63.8)$ & $210(36.2)$ & \\
\hline $7-7.9$ & $1048(76.4)$ & $324(23.6)$ & & $1473(66.3)$ & $749(33.7)$ & \\
\hline $8-8.9$ & $2652(76.4)$ & $818(23.6)$ & & $2928(62.5)$ & $1759(37.5)$ & \\
\hline $9-9.9$ & $2772(77.0)$ & $827(23.0)$ & & $2380(59.6)$ & $1613(40.4)$ & \\
\hline$\geq 10$ & $1942(74.6)$ & $662(25.4)$ & & $1337(57.3)$ & $995(42.7)$ & \\
\hline Nighttime sleep duration (hours) & & & 0.19 & & & 0.005 \\
\hline$<7$ & $599(73.0)$ & $222(27.0)$ & & $738(64.3)$ & $410(35.7)$ & \\
\hline $7-7.9$ & $2346(76.3)$ & $727(23.7)$ & & $2470(63.1)$ & $1445(36.9)$ & \\
\hline $8-8.9$ & $3510(76.4)$ & $1083(23.6)$ & & $3529(60.4)$ & $2316(39.6)$ & \\
\hline$\geq 9$ & $2195(76.1)$ & $688(23.9)$ & & $1751(60.3)$ & $1155(39.7)$ & \\
\hline Daytime napping duration (minutes) & & & 0.08 & & & $<0.001$ \\
\hline None & $2426(77.6)$ & $700(22.4)$ & & $2996(63.9)$ & $1690(27.9)$ & \\
\hline$<30$ & $218(74.9)$ & $73(25.1)$ & & $372(72.1)$ & $144(27.9)$ & \\
\hline $30-59$ & $1281(76.8)$ & $386(23.2)$ & & $1515(17.8)$ & $922(37.8)$ & \\
\hline $60-89$ & $2625(75.6)$ & $849(24.4)$ & & $2303(27.1)$ & $1577(40.6)$ & \\
\hline$\geq 90$ & $2100(74.7)$ & $712(25.3)$ & & $1302(15.3)$ & $993(43.3)$ & \\
\hline
\end{tabular}

Abbreviations: MetS metabolic syndrome. 
Table 3 Adjusted odds ratios (95\% Cl) for metabolic syndrome by sleep duration stratified by gender

\begin{tabular}{|c|c|c|}
\hline & Model 1 & Model 2 \\
\hline \multicolumn{3}{|c|}{ Daily sleep duration (hours) } \\
\hline \multicolumn{3}{|l|}{ Male } \\
\hline$<7$ & $1.21(0.91-1.61)$ & $1.28(0.90-1.80)$ \\
\hline $7-7.9$ & 1.00 & 1.00 \\
\hline $8-8.9$ & $0.99(0.85-1.16)$ & $1.14(0.95-1.37)$ \\
\hline $9-9.9$ & $0.94(0.80-1.09)$ & $1.12(0.93-1.34)$ \\
\hline$\geq 10$ & $1.08(0.92-1.26)$ & $1.10(0.98-1.38)$ \\
\hline \multicolumn{3}{|c|}{ Female } \\
\hline$<7$ & $1.13(0.92-1.39)$ & $1.06(0.84-1.35)$ \\
\hline $7-7.9$ & 1.00 & 1.00 \\
\hline $8-8.9$ & $1.16(1.04-1.30)$ & $1.20(1.06-1.37)$ \\
\hline $9-9.9$ & $1.22(1.08-1.36)$ & $1.28(1.12-1.46)$ \\
\hline$\geq 10$ & $1.29(1.13-1.46)$ & $1.33(1.15-1.54)$ \\
\hline
\end{tabular}

Nighttime sleep duration (hours)

Male

$\begin{array}{llll}<7 & 1.22(1.01-1.46) & 1.15(0.93-1.44) & 1.04(0.93-1.17) \\ 7-7.9 & 1.00 & 1.00 & 1.00 \\ 8-8.9 & 0.99(0.89-1.11) & 1.13(0.99-1.29) & 1.04(0.97-1.12) \\ \geq 9 & 1.01(0.89-1.14) & 1.28(1.10-1.49) & 1.01(0.94-1.10)\end{array}$

Female

$\begin{array}{llll}<7 & 0.96(0.83-1.11) & 0.94(0.79-1.11) & 0.93(0.79-1.10) \\ 7-7.9 & 1.00 & 1.00 & 1.00 \\ 8-8.9 & 1.08(0.99-1.18) & 1.06(0.96-1.17) & 1.05(0.96-1.16) \\ \geq 9 & 1.04(0.93-1.15) & 1.10(0.97-1.24) & 1.10(0.97-1.24)\end{array}$

Daytime napping duration (minutes)

Male

\begin{tabular}{llll} 
None & 1.00 & 1.00 & 1.00 \\
$<30$ & $1.05(0.79-1.40)$ & $0.94(0.67-1.33)$ & $0.94(0.67-1.33)$ \\
$30-59$ & $1.03(0.89-1.19)$ & $1.07(0.90-1.28)$ & $1.07(0.90-1.28)$ \\
$60-89$ & $1.09(0.97-1.23)$ & $1.05(0.91-1.22)$ & $1.05(0.91-1.22)$ \\
$\geq 90$ & $1.12(1.00-1.28)$ & $1.09(0.94-1.27)$ & $1.09(0.94-1.27)$ \\
Female & & & \\
None & 1.00 & 1.00 & 1.00 \\
$<30$ & $0.92(0.73-1.10)$ & $0.87(0.75-1.02)$ & $0.88(0.74-1.03)$ \\
$30-59$ & $1.02(0.92-1.14)$ & $1.03(0.97-1.20)$ & $1.03(0.91-1.16)$ \\
$60-89$ & $1.10(1.00-1.21)$ & $1.08(0.97-1.20)$ & $1.08(0.97-1.20)$ \\
$\geq 90$ & $1.22(1.09-1.36)$ & $1.21(1.06-1.37)$ & $1.20(1.06-1.37)$ \\
\hline
\end{tabular}

Model 1, adjusted for age, marriage, education, smoking status, drinking status, physical activity, coronary heart disease, myocardial infarction, stroke, and family history of hypertension or diabetes.

Model 2, adjusted for the variables in model 1 plus body mass index (BMI). Model 3 , adjusted for the variables in model 2 plus daytime napping duration or nighttime sleep duration, as appropriate. attenuated (OR, 1.20 [95\% CI, 1.06-1.37]). Daily sleep duration, nighttime sleep duration and daytime sleep duration were not associated with MetS among males.

The logistic regression analyses of the relationship between daily sleep duration and the components of MetS showed that long daily sleep duration was independently associated with its components as well except for central obesity in females. Among males, longer daily sleep duration was associated with higher risk of HDL-C criteria only (Table 4).

\section{Discussion}

With a large sample size, we provided epidemiological evidence that long daily sleep duration ( $\geq 8$ hours) is associated with MetS, while short daily sleep duration $(<7$ hours) was not associated with MetS in middle-aged and older Chinese females after adjustment for potential confounders. Moreover, longer daily sleep duration appears to have an adverse effect on the components of the MetS, including triglyceride, HDL-C, blood pressure and glucose. Interestingly, further analysis revealed that longer daytime napping ( $\geq 90$ minutes) rather than longer nighttime sleep is independently associated with a higher risk for MetS.

Few studies have investigated the relationship between the combined sleep duration of nighttime sleep and daytime napping and MetS. The findings of the current study were consistent with one recent study which reported that Korean females who slept $\geq 9 \mathrm{~h} / \mathrm{d}$ had 1.43 times higher risk of MetS compared with those who slept $7 \mathrm{~h} / \mathrm{d}$ [16]. Teresa et al. explored the relationship between total sleep duration and MetS in 29,333 Chinese adults ages $50-96$ years and found that participants who slept longer $(\geq 8 \mathrm{~h} / \mathrm{d})$ were more likely to have MetS and its components [17]. However, Teresa et al. did not revealed gender-specific associations between total sleep duration and MetS or further analyze the relationships among nighttime sleep duration, daytime napping duration and MetS. The reasons for the gender-specific associations are unclear. One explanation may be hormonal differences and the effects of hormones on sleep [18]. Middle-age females progressing the menopausal transition is significantly associated with difficulty sleeping [19]. Older females with decreasing estrogen levels were found to be associated with more trouble falling asleep and frequent awakening [15]. Thus, the association between long sleep duration and MetS may be amplified in females. In addition to the physiological explanation, there have been reported that differential social-economic characteristics and social roles of males and females could lead to the difference of sleep quality [20].

Our findings indicated that neither long nor short nighttime sleep is related to the prevalence of MetS in females and males. The association between nighttime 
Table 4 Adjusted odds ratios of the components of metabolic syndrome by daily sleep duration per day stratified by gender

\begin{tabular}{|c|c|c|c|c|c|}
\hline & \multicolumn{5}{|c|}{ Duration of daily sleep (hours/day) } \\
\hline & $<7$ & $7 \sim$ & $8 \sim$ & $9 \sim$ & $\geq 10$ \\
\hline \multicolumn{6}{|l|}{ Male } \\
\hline Central obesity criterion & $1.26(0.95-1.65)$ & 1.00 & $0.93(0.80-1.07)$ & $0.90(0.80-1.05)$ & $0.89(0.77-1.04)$ \\
\hline Glucose criterion & $0.93(0.71-1.22)$ & 1.00 & $0.92(0.81-1.06)$ & $0.99(0.87-1.15)$ & $1.10(0.96-1.28)$ \\
\hline Blood pressure criterion & $0.87(0.65-1.16)$ & 1.00 & $0.98(0.84-1.13)$ & $0.95(0.82-1.10)$ & $1.05(0.90-1.67)$ \\
\hline HDL-C criterion & $1.26(0.88-1.80)$ & 1.00 & $1.23(1.01-1.49)$ & $1.22(1.01-1.48)$ & $1.40(1.15-1.71)$ \\
\hline Triglycerides criterion & $1.00(0.75-1.35)$ & 1.00 & $0.99(0.85-1.15)$ & $1.12(0.97-1.30)$ & $1.17(0.99-1.36)$ \\
\hline \multicolumn{6}{|l|}{ Female } \\
\hline Central obesity criterion & $1.05(0.85-1.29)$ & 1.00 & $1.11(0.99-1.24)$ & $1.08(0.96-1.21)$ & $1.04(0.91-1.19)$ \\
\hline Glucose criterion & $0.81(0.66-1.00)$ & 1.00 & $0.98(0.88-1.09)$ & $0.96(0.86-1.08)$ & $1.18(1.04-1.34)$ \\
\hline Blood pressure criterion & $1.00(0.80-1.24)$ & 1.00 & $1.08(0.96-1.22)$ & $1.10(0.97-1.24)$ & $1.18(1.02-1.36)$ \\
\hline HDL-C criterion & $0.99(0.79-1.24)$ & 1.00 & $1.02(0.90-1.15)$ & $1.14(1.01-1.29)$ & $1.21(1.05-1.39)$ \\
\hline Triglycerides criterion & $1.07(0.85-1.33)$ & 1.00 & $1.05(0.93-1.18)$ & $1.11(0.98-1.26)$ & $1.15(1.00-1.32)$ \\
\hline
\end{tabular}

Adjust for age, marriage, education, smoking status, drinking status, physical activity, coronary heart disease, myocardial infarction, stroke, and the other four components of metabolic syndrome as appropriate.

$\mathrm{HDL}-\mathrm{C}$, high-density lipoprotein cholesterol.

sleep duration and MetS is controversial. Some studies indicated that both short and long sleep duration is correlated to the risk of MetS, suggesting a U-shaped pattern $[21,22]$. Several studies demonstrated that the risk of MetS increased with short nighttime sleep duration only [23-25]. Another study reported that only long nighttime sleep duration was associated with elevated risk for MetS [26]. There were several possible reasons for observing a nonsignificant relationship between nighttime sleep duration and MetS in our study. First, unlike the studies mentioned above, all the participants of our study were middle-aged and older retired adults who have more time to sleep and less chance for sleep restriction due to retirement. Only $7.8 \%$ of participants slept less than 7 hours per night and only $3.7 \%$ of participants slept less than 6 hours per night, which may have contributed to the lack of power in this study. Second, as for the components of MetS, we found that longer nighttime sleep duration was a risk factor for reduced HDL-cholesterol and elevated triglycerides, while it was a protective factor for central obesity (data not shown). This opposing direction of the associations between nighttime sleep duration and the components of MetS might be one explanation for the absence of a relationship between nighttime sleep duration and MetS.

Few studies have examined the association between daytime napping and MetS. Lin et al. found that a longer daytime napping duration but not a longer nighttime sleeping duration was associated with a higher risk for MetS in females, which was consistent with our findings [12]. Compared with the study of Lin et al., our study has the strength of large sample size and abundant covariates.
Data from 16,480 older adults from the Guangzhou Biobank Cohort Study revealed that a higher frequency of daytime napping was independently related to an increased risk of MetS [11]. However, they assessed the frequency of daytime napping rather than the duration of it. The mechanism underlying the association between longer daytime napping duration and MetS is unclear. Sleep could have various influences on metabolism and endocrine secretion patterns by interfering with the circadian rhythm [27,28]. Circadian rhythm disturbances arising from excessive daytime napping could be one explanation for the relationship between longer daytime napping duration and MetS. Circadian rhythm disturbances can lead to insulin resistance, which plays a major role in the development of MetS and is almost always present with other metabolic abnormalities [29,30]. Another physiologic pathway which plays a role in the relationship between daytime napping and MetS may be sympathetic activation [31]. It has been reported that the practice of napping can induce the elevation of blood pressure mediated by sympathetic activation [32]. Indeed, the sympathetic nervous system is crucial in circulatory and metabolic control and was found to be associated with MetS [33]. Previous studies have reported that long daytime sleep duration was associated with an increased risk for cardiovascular mortality in the elderly $[10,34]$. The presence of MetS carries an elevated risk of cardiovascular disease [3]. Therefore, our results may provide a mechanism linking longer daytime sleep duration and cardiovascular mortality via mediation of MetS.

Several potential limitations of our study need to be mentioned. First, we collected the information about 
sleep duration based on self-reports. Despite high correlations between subjective estimates of sleep duration and more direct assessments such as actigraphy [35,36], other objective measurements of sleep duration are needed to support our findings. Secondly, the crosssectional design limits causal inferences on the association between sleep duration and MetS. However, in the Chinese context, daytime napping is considered healthy and most Chinese develop the habit of daytime napping in youth [37], making the reverse association unlikely in this case. Third, we did not include other potential confounders that could affect sleep patterns such as obstructive sleep apnea (OSA). OSA is independently correlated with insulin resistance and a high risk of MetS, and is known to cause excessive sleepiness [38,39], with habitual nappers having a higher frequency of OSA [40]. Missing data on these crucial factors could inevitably lead to potential bias in the evaluation of the odds ratios for MetS. Finally, this study was performed in a Chinese population, and our results might not be able to be generalized to other races and ethnic groups.

The strengths of our study include a large sample size; the availability of blood measurements; the abundant data on potential confounding factors; and standardized questionnaires, which not only enhance the precision but also allow statistical adjustment for multiple variables.

\section{Conclusions}

Longer daytime napping duration rather than nighttime sleep duration is associated with a moderate elevated risk of MetS in females. The findings have significant implications for further study to examine the appropriate duration of sleep for older adults especially for females.

\section{Competing interests}

The authors declared that they have no competing interest.

\section{Authors' contributions}

JW and YW conceived and designed the study; GX, LS, YZ and SY performed the statistical analysis; HY and TW performed the experiments; JW drafted the article; YW and YL contributed to the critical revision of the article. All authors gave their comments on the article and approved the final version.

\section{Acknowledgments}

This research was funded by the National Natural Science Foundation of China (81273083), the Fundamental Research Funds for the Central Universities (2014TS051), the 111 project, the Program for Changjiang Scholars, Innovative Research Team in University, China Medical Board 12-113. We thank the Tongji-Dongfeng Cohort participants and the staff at DMC in Dongfeng General Hospital for the data collection.

\footnotetext{
Author details

${ }^{1}$ MOE Key Lab of Environment and Health, School of Public Health, Tongji Medical College, Huazhong University of Science \& Technology, Hangkong Road 13, Wuhan 430030, Hubei, China. ${ }^{2}$ Department of Maternal and Child Health, School of Public Health, Tongji Medical College, Huazhong University of Science \& Technology, Hangkong Road 13, Wuhan 430030, Hubei, China. ${ }^{3}$ Dongfeng General Hospital, Dongfeng Motor Corporation and Hubei University of Medicine, Daling Road 16, Shiyan 442000, Hubei, China. ${ }^{4}$ Department of Social Medicine, School of Public Health, Tongji Medical
}

College, Huazhong University of Science \& Technology, Hangkong Road 13, Wuhan 430030, Hubei, China.

Received: 11 November 2014 Accepted: 11 February 2015

Published online: 24 February 2015

\section{References}

1. Alberti KG, Zimmet $P$, Shaw J. The metabolic syndrome-a new worldwide definition. Lancet. 2005;366(9491):1059-62

2. Chen J, Muntner P, Hamm LL, Jones DW, Batuman V, Fonseca $V$, et al. The metabolic syndrome and chronic kidney disease in US adults. Ann Intern Med. 2004;140(3):167-74.

3. McNeill AM, Rosamond WD, Girman CJ, Golden SH, Schmidt MI, East HE et al. The metabolic syndrome and 11-year risk of incident cardiovascular disease in the atherosclerosis risk in communities study. Diabetes Care. 2005;28(2):385-90.

4. Ford ES. The metabolic syndrome and mortality from cardiovascular disease and all-causes: findings from the National Health and Nutrition Examination Survey II Mortality Study. Atherosclerosis. 2004;173(2):307-12.

5. Gu D, Reynolds K, Wu X, Chen J, Duan X, Reynolds RF, et al. Prevalence of the metabolic syndrome and overweight among adults in China. Lancet. 2005:365(9468):1398-405.

6. Ju SY, Choi WS. Sleep duration and metabolic syndrome in adult populations: a meta-analysis of observational studies. Nutr Diabetes. 2013;3:e65.

7. Floyd JA, Medler SM, Ager JW, Janisse JJ. Age-related changes in initiation and maintenance of sleep: a meta-analysis. Res Nurs Health. 2000;23(2):106-17.

8. Ohayon MM, Carskadon MA, Guilleminault C, Vitiello MV. Meta-analysis of quantitative sleep parameters from childhood to old age in healthy individuals: developing normative sleep values across the human lifespan. Sleep. 2004;27:1255-74

9. Liu L, Tang J, Liu X, Liu Z, Zhao G, Wang G, et al. Sleep behavior of the urban elderly: a cross-sectional study. Chin J Behav Med Sci. 2002;11(3):310-2 [In Chinese].

10. Leng Y, Wainwright NW, Cappuccio FP, Surtees PG, Hayat S, Luben R, et al. Daytime napping and the risk of All-cause and cause-specific mortality: a 13-year follow-up of a British population. Am J Epidemiol. 2014;179(9):1115-24.

11. Xu Q, Song Y, Hollenbeck A, Blair A, Schatzkin A, Chen H. Day napping and short night sleeping are associated with higher risk of diabetes in older adults. Diabetes Care. 2010;33(1):78-83.

12. Lin D, Sun K, Li F, Qi Y, Ren M, Huang C, et al. Association between habitual daytime napping and metabolic syndrome: a population-based study. Metabolism. 2014;63(12):1520-7

13. Wang F, Zhu J, Yao P, Li X, He M, Liu Y, et al. Cohort Profile: the DongfengTongji cohort study of retired workers. Int J Epidemiol. 2013;42(3):731-40.

14. Belfki H, Ali SB, Aounallah-Skhiri H, Traissac P, Bougatef S, Maire B, et al. Prevalence and determinants of the metabolic syndrome among Tunisian adults: results of the transition and health impact in North Africa (TAHINA) project. Public Health Nutr. 2013;16(04):582-90.

15. Newman AB, Enright PL, Manolio TA, Haponik EF. Sleep disturbance, psychosocial correlates, and cardiovascular disease in 5201 older adults: the cardiovascular health study. J Am Geriatr Soc. 1997;45(1):1-7.

16. Stefani KM, Kim HC, Kim J, Oh K, Suh I. The influence of sex and age on the relationship between sleep duration and metabolic syndrome in Korean adults. Diabetes Res Clin Pract. 2013;102(3):250-9.

17. Arora T, Jiang CQ, Thomas GN, Lam K-bH, Zhang WS, Cheng KK, et al. Self-Reported long total sleep duration is associated with metabolic syndrome the Guangzhou Biobank Cohort Study. Diabetes Care. 2011;34(10):2317-9.

18. Krishnan V, Collop NA. Gender differences in sleep disorders. Cur Opin Pulm Med. 2006;12(6):383-9.

19. Kravitz HM, Zhao X, Bromberger JT, Gold EB, Hall MH, Matthews KA, et al Sleep disturbance during the menopausal transition in a multi-ethnic community sample of women. Sleep. 2008;31(7):979.

20. Arber S, Bote M, Meadows R. Gender and socio-economic patterning of self-reported sleep problems in Britain. Soc Sci Med. 2009;68(2):281-9.

21. Choi K, Lee J, Park H, Baik S, Choi D, Kim S. Relationship between sleep duration and the metabolic syndrome: Korean National Health and Nutrition Survey 2001. Int J Obes. 2008;32(7):1091-7.

22. Ohkuma T, Fujii H, Iwase M, Ogata-Kaizu S, Ide H, Kikuchi Y, et al. U-shaped association of sleep duration with metabolic syndrome and insulin resistance in patients with type 2 diabetes: the Fukuoka diabetes registry. Metabolism. 2014;63(4):484-91. 
23. Wu MC, Yang YC, Wu JS, Wang RH, Lu FH, Chang CJ. Short sleep duration associated with a higher prevalence of metabolic syndrome in an apparently healthy population. Prev Med. 2012;55(4):305-9.

24. Kobayashi D, Takahashi O, Deshpande GA, Shimbo T, Fukui T. Relation between metabolic syndrome and sleep duration in Japan: a large scale cross-sectional study. Intern Med. 2011;50(2):103-7.

25. Otsuka T, Kawada T, Yanai M, Kitagawa Y, Kan H. Incidence of metabolic syndrome and associated lifestyle factors in a worksite male population. J Occup Health. 2011;53(3):78-86.

26. Santos A-C, Ebrahim S, Barros H. Alcohol intake, smoking, sleeping hours, physical activity and the metabolic syndrome. Prev Med. 2007;44(4):328-34

27. Morris CJ, Aeschbach D, Scheer FA. Circadian system, sleep and endocrinology. Mol Cell Endocrinol. 2012;349(1):91-104.

28. Ando H. Circadian clocks and lifestyle-related diseases. Jpn J Clin Pathol. 2013;61(11):1044-50.

29. Shi SQ, Ansari TS, McGuinness OP, Wasserman DH, Johnson CH. Circadian disruption leads to insulin resistance and obesity. Curr Biol. 2013;23(5):372-81 [In Japanese].

30. Bonora E, Kiechl S, Willeit J, Oberhollenzer F, Egger G, Targher G, et al. Prevalence of insulin resistance in metabolic disorders: the Bruneck Study. Diabetes. 1998;47(10):1643-9.

31. Mancia G, Bousquet P, Elghozi JL, Esler M, Grassi G, Julius S, et al. The sympathetic nervous system and the metabolic syndrome. J Hypertens. 2007;25(5):909-20.

32. Mulcahy D, Wright C, Sparrow J, Cunningham D, Curcher D, Purcell H, et al. Heart rate and blood pressure consequences of an afternoon SIESTA (Snooze-Induced Excitation of Sympathetic Triggered Activity). Am J Cardiol. 1993;71(7):611-4.

33. Lambert GW, Straznicky NE, Lambert EA, Dixon JB, Schlaich MP. Sympathetic nervous activation in obesity and the metabolic syndrome-causes, consequences and therapeutic implications. Pharmacol Ther. 2010;126(2):159-72.

34. Tanabe N, Iso H, Seki N, Suzuki H, Yatsuya H, Toyoshima H, et al. Daytime napping and mortality, with a special reference to cardiovascular disease: the JACC study. Int J Epidemiol. 2010;39(1):233-43.

35. Signal TL, Gale J, Gander PH. Sleep measurement in flight crew: comparing actigraphic and subjective estimates to polysomnography. Aviat Space Environ Med. 2005;76(11):1058-63.

36. Lockley SW, Skene DJ, Arendt J. Comparison between subjective and actigraphic measurement of sleep and sleep rhythms. J Sleep Res. 1999;8(3):175-83.

37. RH YY, Li X, Wang B, Kong L, Li D, Bao W. Survey on napping among college students. Chin J Clin Rehabil. 2006;10(22):43-5 [In Chinese]

38. Coughlin SR, Mawdsley L, Mugarza JA, Calverley PM, Wilding JP. Obstructive sleep apnoea is independently associated with an increased prevalence of metabolic syndrome. Eur Heart J. 2004;25(9):735-41.

39. Gottlieb DJ, Whitney CW, Bonekat WH, Iber C, James GD, Lebowitz M, et al. Relation of sleepiness to respiratory disturbance index: the sleep heart health study. Am J Respir Crit Care Med. 1999;159(2):502-7.

40. Masa JF, Rubio M, Perez P, Mota M, de Cos JS, Montserrat JM. Association between habitual naps and sleep apnea. Sleep. 2006;29(11):1463.

\section{Submit your next manuscript to BioMed Central and take full advantage of:}

- Convenient online submission

- Thorough peer review

- No space constraints or color figure charges

- Immediate publication on acceptance

- Inclusion in PubMed, CAS, Scopus and Google Scholar

- Research which is freely available for redistribution 